Kalpa Publications in Computing
Volume 2, 2017, Pages $135-144$
ICRISET2017. International Conference on Re-
search and Innovations in Science, Engineer-
ing \&Technology. Selected Papers in Computing

\title{
A Numerical Solution of Uni-Dimensional Vertical Ground Water Recharge through Porous Media by Finite Difference Method Using Matlab
}

\author{
Tejas B. Shah ${ }^{1}$ and Dr. Manish R. Tailor ${ }^{2}$ \\ ${ }^{1}$ Research scholar, Department of Mathematics, Veer Narmad South Gujarat UniversitySurat, \\ India. \\ ${ }^{2}$ Asst. professor, Department of Mathematics, The Patidar Gin Science College, Bardoli, India. \\ shah.tejas16988@gmail.com, mtailor21@gmail.com
}

\begin{abstract}
This paper shows an approximate solution of Uni-dimensional vertical ground water recharge that can be solved using finite difference method with matlab programme. The average diffusivity coefficient over the whole range of moisture content is regarded as constant and a parabolic variation of permeability with moisture content is assumed.
\end{abstract}

Key words

Porous media, Single phase fluid, Finite Difference method, MATLAB Programme.

\section{INTRODUCTION}

The problem of flow of water through partially saturated porous media has been discussed by Klutte (1952) [2], Sharma (1965) [5], Verma (1969) [6, 7], Patel (1997) [4]. The first two authors have been employed a numerical method to investigate it while the uses a transformation technique to analyse behaviour. The fourth author employs Whittaker's confluent hypergeometric function. The fifth author obtained the solution of this problem by Laplace transform technique. In present paper we have obtained solution of the problem by using finite difference method, Grewal B.S. [1].

In the present mathematical model we consider that the ground water recharge takes place over the large basin of such geological location whose sides are limited by rigid boundaries and the bottom by a thick layer of water table. In this case we assumed that the flows are vertically downward through unsaturated porous media. Here, the average diffusivity coefficient of the whole range of moisture content is regarded as constant (Mehta, 1977) [3] and the permeability of the moisture content is assumed to a linear function of moisture content (Patel, 1997) [4]. The theoretical formulation of the problem gives a nonlinear partial differential equation for the moisture content. 


\section{FORMULATION OF THE PROBLEM}

The equation of continuity for an unsaturated medium is given by

$$
\frac{\partial}{\partial t}\left(\rho_{s} \theta\right)=-\nabla M
$$

Where, $\rho_{s}$ is the bulk density of the medium, $\theta$ is its moisture content on a dry weight basis and $M$ is the mass flux moisture.

From Darcy's law for the motion in a porous medium, we get

$$
V=-\mathrm{K} \nabla \varphi
$$

Where, $\nabla \varphi$ represent the gradient of the whole moisture potential, $V$ is the volume flux of moisture and $K$ is the coefficient of aqueous conductivity.

Combining equation (2.1) and (2.2) we obtain

$$
\frac{\partial}{\partial t}\left(\rho_{s} \theta\right)=\nabla(\rho K \nabla \varphi)
$$

Where, $\rho$ is the fluid density, as in the present case we consider that the flow takes place only in the vertical direction, equation (2.3) reduces to

$$
\rho_{s} \frac{\partial \theta}{\partial t}=\frac{\partial}{\partial z}\left(\rho K \frac{\partial \Psi}{\partial z}\right)-\frac{\partial}{\partial z}(\rho K g)
$$

Where, $\Psi$ is the pressure (capillary) potential, $g$ is the gravitational content and $\varphi=\Psi-g z$. The positive direction of ' $Z$-axis' is the same as that of the gravity.

Considering $\theta$ and $\Psi$ to be connected by single valued function, we may write (2.4) as

$$
\frac{\partial \theta}{\partial t}=\frac{\partial}{\partial z}\left(D \frac{\partial \theta}{\partial z}\right)-\frac{\rho}{\rho_{s}} g \frac{\partial K}{\partial z}
$$

Where $D=\frac{\rho}{\rho_{s}} K \frac{\partial \Psi}{\partial z}$ and is called diffusivity coefficient.

We can replace $D$ by its average value $D_{a}$ and assuming $K=K_{0} \theta,\left(K_{0}=0.232\right)$ where $K_{0}$ is constant, we have

$$
\frac{\partial \theta}{\partial t}=D_{a} \frac{\partial^{2} \theta}{\partial z^{2}}-\frac{\rho}{\rho_{s}} K_{0} \frac{\partial \theta}{\partial z}
$$

Considering the water table to be situated at a depth $L$, and putting

$$
\frac{Z}{L}=\xi, \frac{t D_{a}}{L^{2}}=T
$$

We may write the differential equation (2.6) in the form

$$
\frac{\partial \theta}{\partial T}=\frac{\partial^{2} \theta}{\partial \xi^{2}}-\frac{\rho}{\rho_{s}} \frac{K_{0}}{D_{a}} \frac{\partial \theta}{\partial \xi}
$$

It may be mentioned for definiteness that a set of appropriate boundary condition is

$$
\theta(0, T)=\theta_{0}, \theta(1, T)=1, \theta(\xi, 0)=\theta_{\varepsilon}<<1
$$

Where the moisture content throughout the region is regarded as constant and initially at the large $Z=1$, it reaches up to $100 \%$. 


\section{SOLUTION BY FINITE DIFFERENCE METHOD}

Putting $\frac{\rho}{\rho_{s}} \frac{K_{0}}{D_{a}}=\beta$ (constant) in equation (2.7) we get,

$$
\frac{\partial \theta}{\partial T}=\frac{\partial^{2} \theta}{\partial \xi^{2}}-\beta \frac{\partial \theta}{\partial \xi}
$$

With $\theta(0, T)=\theta_{0}, \theta(1, T)=1, \theta(\xi, 0)=\theta_{\varepsilon}<<1$

Consider finite difference standard five point formula Let

$$
\begin{aligned}
& \theta(\xi, T)=U_{i, j} \\
& \theta_{\xi}=\frac{U_{i, j}-U_{i-1, j}}{h}+o(h) \\
& \theta_{\xi \xi}=\frac{U_{i-1, j}-2 U_{i, j}+U_{i+1, j}}{h^{2}}+o\left(h^{2}\right) \\
& \theta_{T}=\frac{U_{i, j}-U_{i, j-1}}{k}+o(k)
\end{aligned}
$$

Consider a rectangular region $\mathrm{R}$ for which $\theta(\xi, T)$ is known at the boundary. Divide this region in to a network of square mash of side h, as shown in Figurel (Assuming that an exact subdivision of $\mathrm{R}$ is possible). Replacing that derivatives in equation number (3.1) by their difference approximations, we have

$$
\frac{1}{h^{2}}\left(U_{i-1, j}-2 U_{i, j}+U_{i+1, j}\right)-\frac{\beta}{h}\left(U_{i, j}-U_{i-1, j}\right)-\frac{1}{k}\left(U_{i, j}-U_{i, j-1}\right)=0
$$

Equation number (3.6) is the finite difference scheme for the governing partial differential equation. This is exhibited in Figure 1 given below.

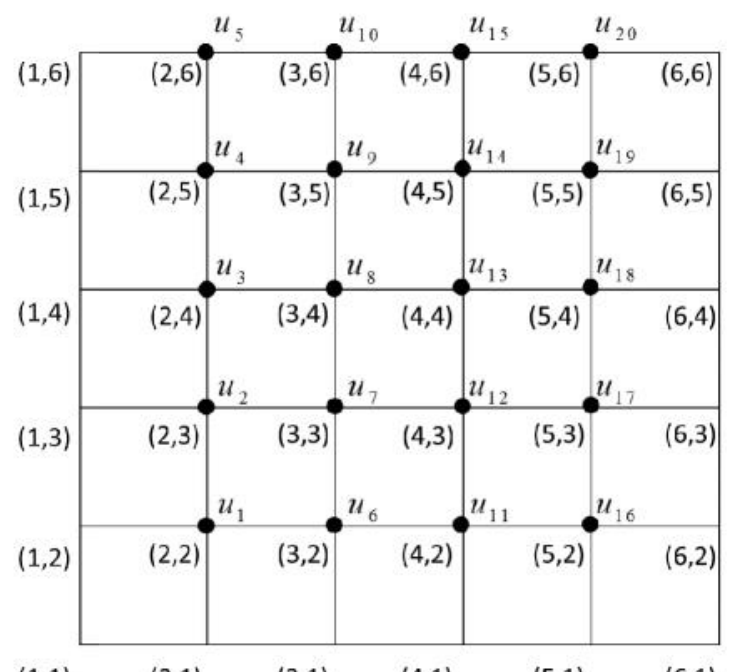

Figure 1 
Now take

$$
\begin{aligned}
& n=5, h=\frac{b-a}{n}=k \\
& h=0.2, k=0.2
\end{aligned}
$$

That gives the nodes $u_{1}$ to $u_{20}$. For all $(i, j)$ it can be defined shown as following.

$\begin{array}{lllll}u_{1}=U_{2,2} & u_{5}=U_{2,6} & u_{9}=U_{3,5} & u_{13}=U_{4,4} & u_{17}=U_{5,3} \\ u_{2}=U_{2,3} & u_{6}=U_{3,2} & u_{10}=U_{3,6} & u_{14}=U_{4,5} & u_{18}=U_{5,4} \\ u_{3}=U_{2,4} & u_{7}=U_{3,3} & u_{11}=U_{4,2} & u_{15}=U_{4,6} & u_{19}=U_{5,5} \\ u_{4}=U_{2,5} & u_{8}=U_{3,4} & u_{12}=U_{4,3} & u_{16}=U_{5,2} & u_{20}=U_{5,6}\end{array}$

From the equation number (3.6), we can write the equation for all $(i, j)$ by taking $\theta_{0}=0.001, \theta_{\varepsilon}=0.01$, and $\beta=0.00001$

We get,

$$
\begin{aligned}
& -u_{1}+0.4288 u_{6}=-0.0018 \\
& 0.1415 u_{1}-u_{2}+0.4288 u_{7}=-0.0004 \\
& 0.1415 u_{2}-u_{3}+0.4288 u_{8}=-0.0004 \\
& 0.1415 u_{3}-u_{4}+0.4288 u_{9}=-0.0004 \\
& 0.1415 u_{4}-u_{5}+0.4288 u_{10}=-0.0004 \\
& 0.4288 u_{1}-u_{6}+0.4288 u_{11}=-0.0014 \\
& 0.4288 u_{2}+0.1415 u_{6}-u_{7}+0.4288 u_{12}=0 \\
& 0.4288 u_{3}+0.1415 u_{7}-u_{8}+0.4288 u_{13}=0 \\
& 0.4288 u_{4}+0.1415 u_{8}-u_{9}+0.4288 u_{14}=0 \\
& 0.4288 u_{5}+0.1415 u_{9}-u_{10}+0.4288 u_{15}=0 \\
& 0.4288 u_{6}-u_{11}+0.4288 u_{16}=-0.0014 \\
& 0.4288 u_{7}+0.1415 u_{11}-u_{12}+0.4288 u_{17}=0 \\
& 0.4288 u_{8}+0.1415 u_{12}-u_{13}+0.4288 u_{18}=0 \\
& 0.4288 u_{9}+0.1415 u_{13}-u_{14}+0.4288 u_{19}=0 \\
& 0.4288 u_{10}+0.1415 u_{14}-u_{15}+0.4288 u_{20}=0 \\
& 0.4288 u_{11}-u_{16}=-0.4302 \\
& 0.4288 u_{12}+0.1415 u_{16}-u_{17}=-0.4288 \\
& 0.4288 u_{13}+0.1415 u_{17}-u_{18}=-0.4288 \\
& 0.4288 u_{14}+0.1415 u_{18}-u_{19}=-0.4288 \\
& 0.4288 u_{15}+0.1415 u_{19}-u_{20}=-0.4288
\end{aligned}
$$

Equation number (3.7) to (3.26) gives the system. Solving that system by simply the inverse method, we get the solution for the nodes $u_{1}$ to $u_{20}$.

That is given by 


$$
\begin{array}{lllll}
u_{1}=0.0743 & u_{5}=0.1917 & u_{9}=0.3718 & u_{13}=0.5397 & u_{17}=0.7106 \\
u_{2}=0.1319 & u_{6}=0.1690 & u_{10}=0.3856 & u_{14}=0.5708 & u_{18}=0.7608 \\
u_{3}=0.1659 & u_{7}=0.2823 & u_{11}=0.3165 & u_{15}=0.5849 & u_{19}=0.7812 \\
u_{4}=0.1833 & u_{8}=0.3425 & u_{12}=0.4705 & u_{16}=0.5659 & u_{20}=0.7902
\end{array}
$$

Figure 2 and Figure 3 show the 3D surface for solution of the problem form different sides.

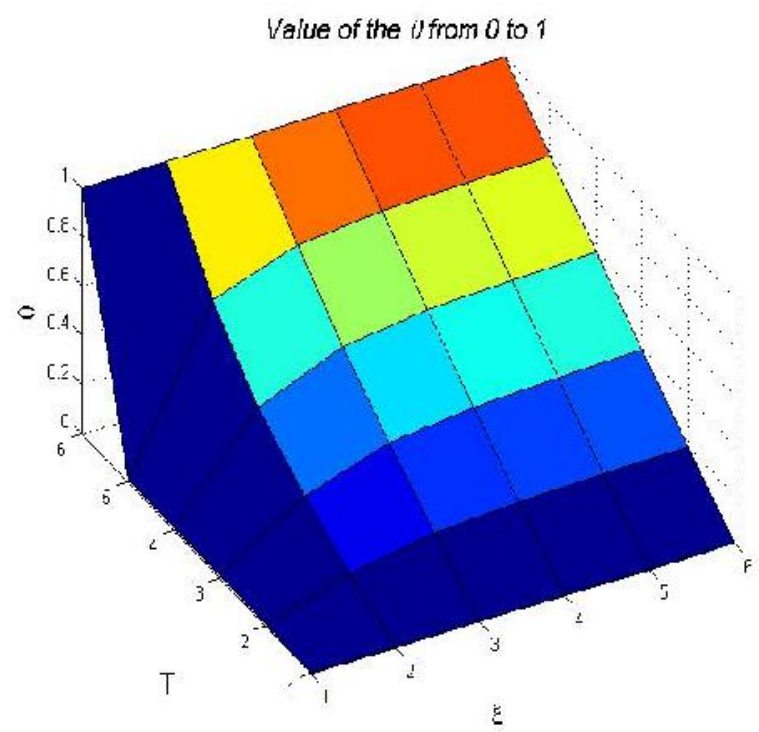

Figure 2

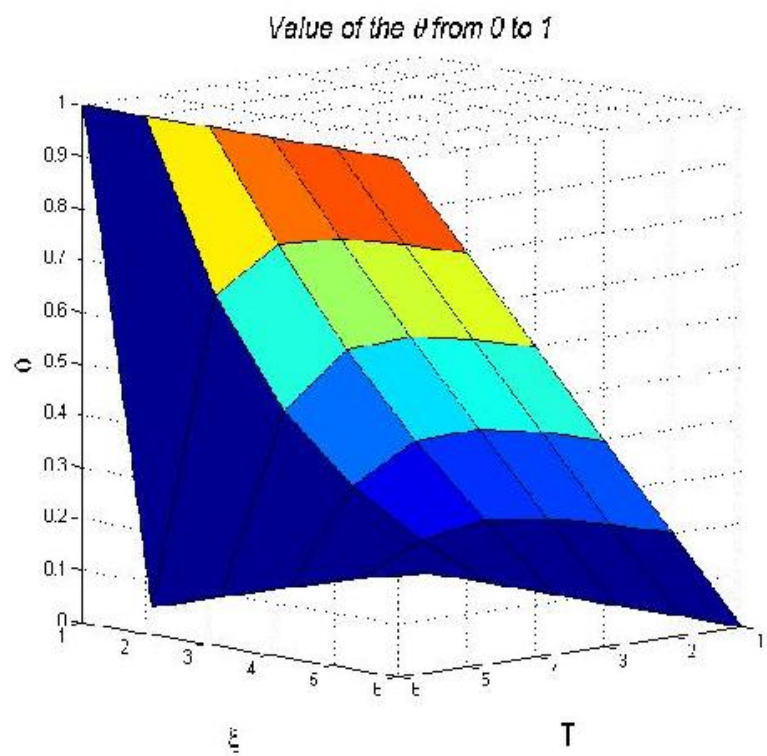

Figure 3 


\section{CONCLUSION}

Value of the moisture content $\theta$ at different points of $\xi$ and $T$ are given in the following table.

TABLE 1 , we fix " $\xi$ " and find the value of " $\theta$ " for the different value of " $T$ ". Whose graph is defined in the Figure 4.

\begin{tabular}{|c|c|c|c|c|c|c|}
\hline \multirow{2}{*}{$\begin{array}{c}\text { VALUE } \\
\text { OF } \boldsymbol{0}\end{array}$} & \multicolumn{6}{|c|}{ VALUE OF $\boldsymbol{0}$} \\
\cline { 2 - 7 } & $\mathbf{0}$ & $\mathbf{0 . 2}$ & $\mathbf{0 . 4}$ & $\mathbf{0 . 6}$ & $\mathbf{0 . 8}$ & $\mathbf{1}$ \\
\hline $\mathbf{0}$ & 0.001 & 0.01 & 0.01 & 0.01 & 0.01 & 1 \\
\hline $\mathbf{0 . 2}$ & 0.001 & 0.0743 & 0.169 & 0.3165 & 0.5659 & 1 \\
\hline $\mathbf{0 . 4}$ & 0.001 & 0.1319 & 0.2823 & 0.4705 & 0.7106 & 1 \\
\hline $\mathbf{0 . 6}$ & 0.001 & 0.1659 & 0.3425 & 0.5397 & 0.7608 & 1 \\
\hline $\mathbf{0 . 8}$ & 0.001 & 0.1833 & 0.3718 & 0.5708 & 0.7812 & 1 \\
\hline $\mathbf{1}$ & 0.001 & 0.1917 & 0.3856 & 0.5849 & 0.7902 & 1 \\
\hline
\end{tabular}

TABLE 1

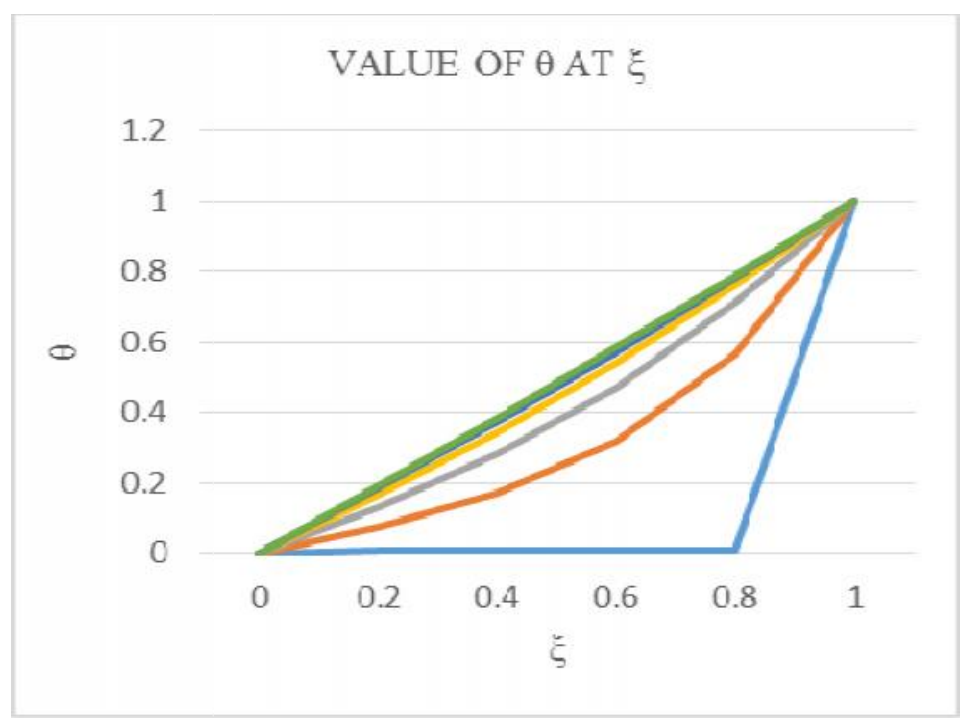

Figure 4

TABLE 2, we fix " $T$ " and find the value of " $\theta$ " for the different value of " $\xi$ ". Whose graph is defined in the Figure 5. 


\begin{tabular}{|c|c|c|c|c|c|c|}
\hline \multirow{2}{*}{$\begin{array}{c}\text { VALUE } \\
\text { OF } \boldsymbol{\theta}\end{array}$} & \multicolumn{7}{|c|}{ VALUE AT T } \\
\cline { 2 - 7 } & $\mathbf{0}$ & $\mathbf{0 . 2}$ & $\mathbf{0 . 4}$ & $\mathbf{0 . 6}$ & $\mathbf{0 . 8}$ & $\mathbf{1}$ \\
\hline $\mathbf{0}$ & 0.001 & 0.001 & 0.001 & 0.001 & 0.001 & 0.001 \\
\hline $\mathbf{0 . 2}$ & 0.01 & 0.0743 & 0.1319 & 0.1659 & 0.1833 & 0.1917 \\
\hline $\mathbf{0 . 4}$ & 0.01 & 0.169 & 0.2823 & 0.3425 & 0.3718 & 0.3856 \\
\hline $\mathbf{0 . 6}$ & 0.01 & 0.3165 & 0.4705 & 0.5397 & 0.5708 & 0.5849 \\
\hline $\mathbf{0 . 8}$ & 0.01 & 0.5659 & 0.7106 & 0.7608 & 0.7812 & 0.7902 \\
\hline $\mathbf{1}$ & 1 & 1 & 1 & 1 & 1 & 1 \\
\hline
\end{tabular}

TABLE 2

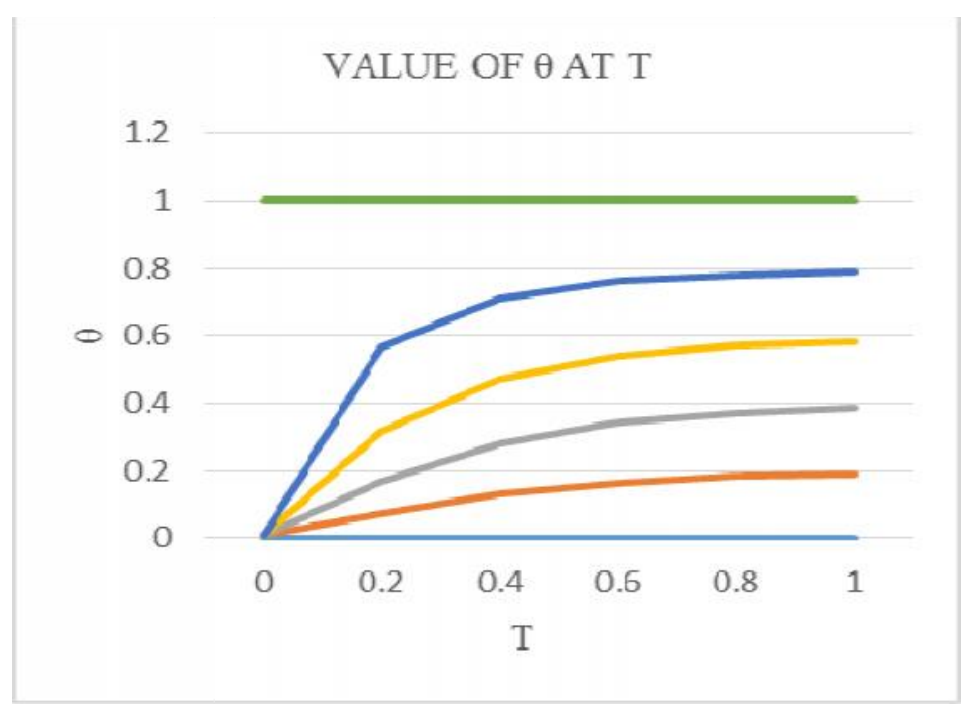

Figure 5

Now we can more generalize the problem by making more iteration and the solution is clear. For that I used MATLAB programming and get the nearest solution of the problem.

\section{MATLAB PROGRAMME OF THE PROBLEM}

Here, We define the general MATLAB programming for the given problem in which by guessing very small step size (the value of $\mathbf{m}$ and $\mathbf{n}$ ) we get very small value of $\mathbf{h}$ and $\mathbf{k}$. that means increasing the meshes gives the large number of nodes (grid points). Also we can do the same for the different boundary and the result will be clear for us.

The MATLAB programme of the problem is given by 


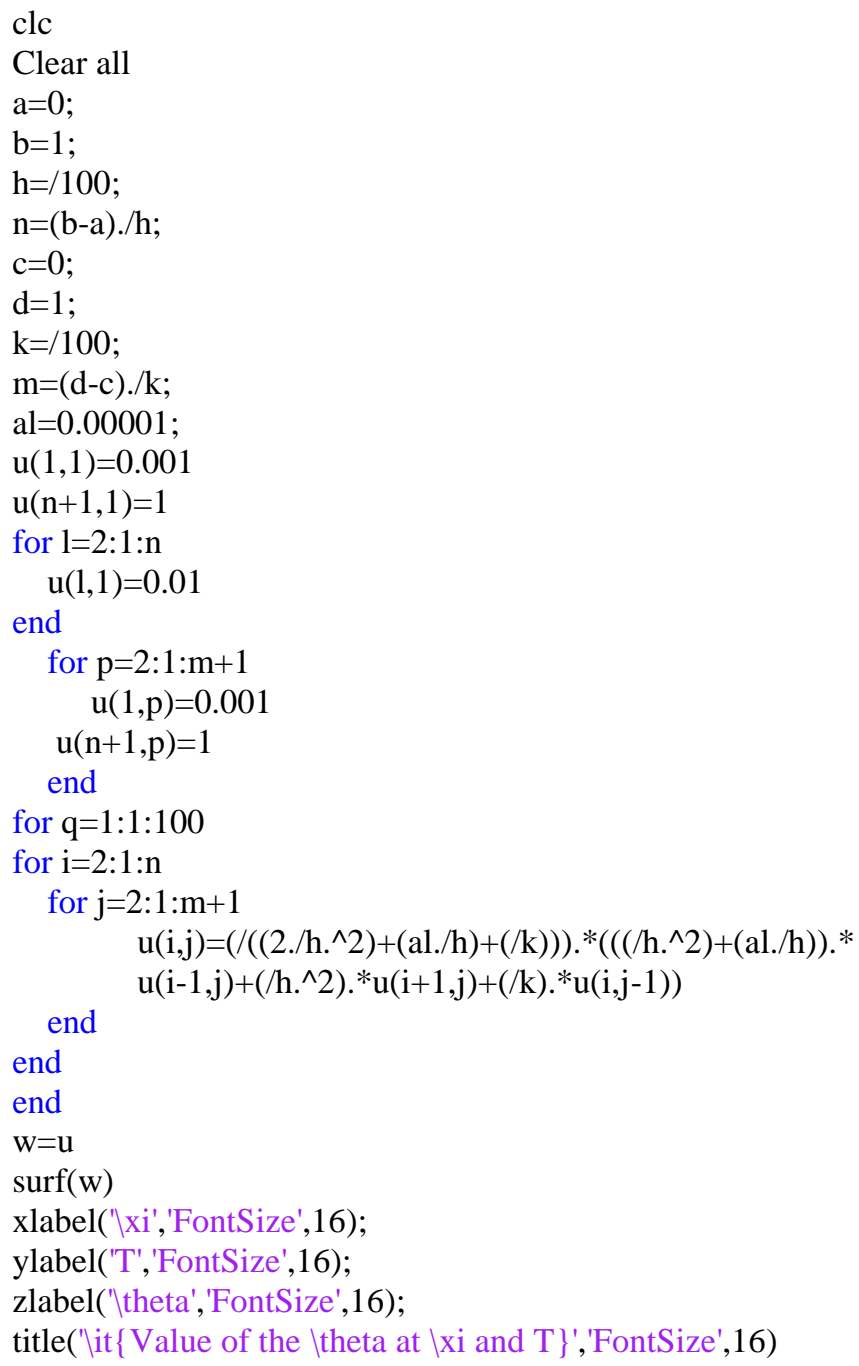

This is the programme for the given problem in which $(a, b)$ and $(c, d)$ denotes the boundary points for the mesh, $\mathrm{n}$ and $\mathrm{m}$ is the number of mesh you taken which gives the value of $\mathrm{h}$ and $\mathrm{k}$ means length between the grid points respectively. And boundary value also defined. Also here we assumed the value of al= $\beta$. q Defines the number of iteration, by taking large number of iteration we get the nearer solution of the problem. W gives the final value of the nodes $u_{i}$.

Now I take the large value of $\mathrm{m}=100$ and $\mathrm{n}=100$ gives the small value of $\mathrm{h}=0.01$ and $\mathrm{k}=0.01$ respectively. This gives the total 9900 nodes means $u_{1}$ to $u_{9900}$. Whose values are find using MATLAB. For that the solution is defined in the following Figures Figure 6 shows the 2D- plot for the solution of the problem. Figure 7 and Figure 8 that shows the 3D surface for the solution of the problem from different sides. 


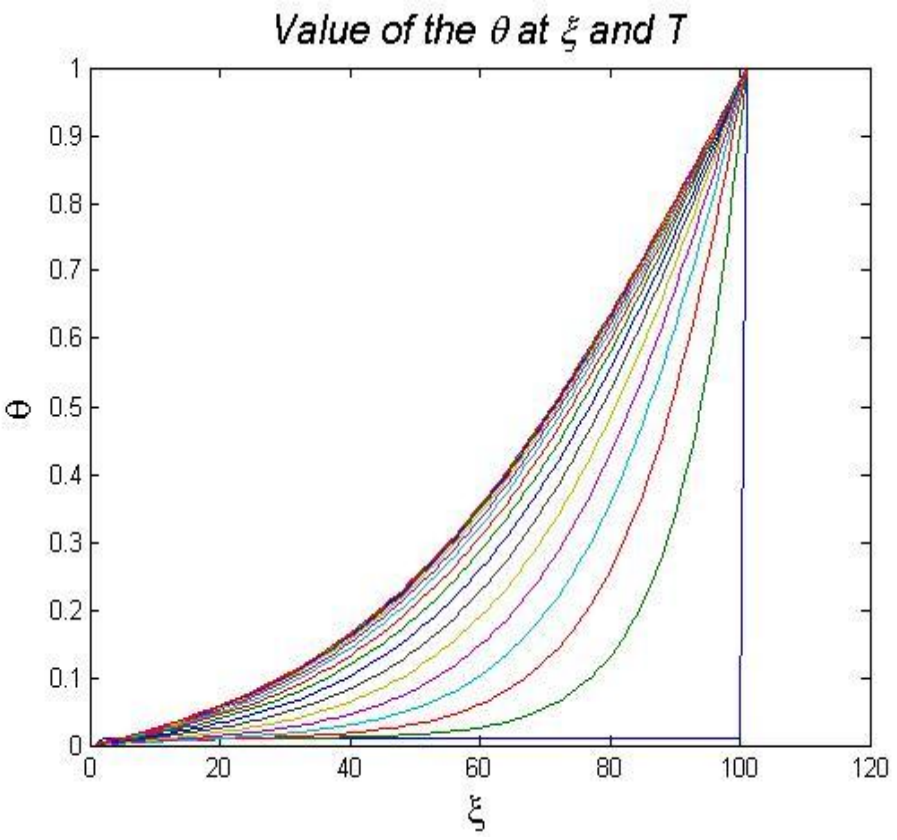

Figure 6

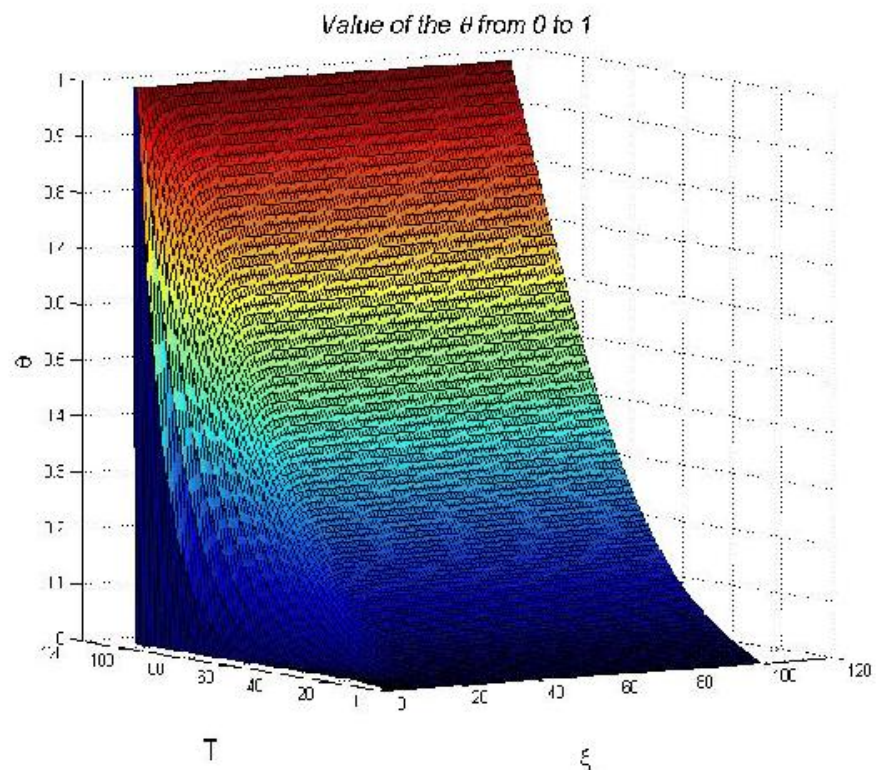

Figure 7 


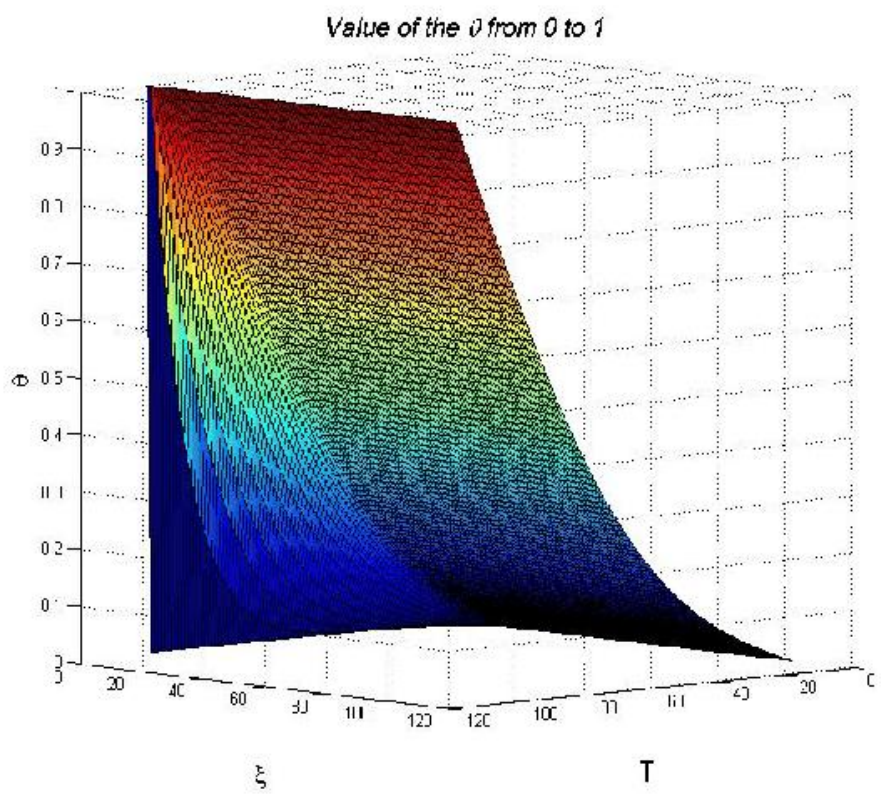

Figure 8

\section{REFERENCES}

[1] Grewal, B.S. (2010): Numerical methods in engineering and science, Khanna publishers, ISBN-13, 978-8174092489.

[2] Klute, A. (1952): A numerical method for solving the flow equation for water in unsaturated materials. Soil science, 73 (2); p.105.

[3] Mehta, M.N. (1977): Asymptotic expansion of fluid flow through porous media; Ph.D. thesis, south Gujarat University, Surat.

[4] Patel, S. S. (1997): An approximate solution of displacement processes in porous media, Ph.D. thesis, south Gujarat University, Surat.

[5] Sharma, S. V. K. (1965). Problem of partially saturated unsteady state of flow through groundwater recharge by spearing, J. Sci. Engineering Recharge, 9; p.69.

[6] Verma, A.P. (1969): Rev. Roum. Sci. Techn. Ser. Mech. Appli., 14,471.

[7] Verma, A.P. (1969): The laplace transform solution of one dimensional ground water recharge by spreading annali di Geofiica; XXII-1; 25. 